\title{
BEAM LOSS AT THE BEGINNING OF ACCELERATION IN THE KEK-PS MAIN RING
}

\author{
T. Toyama, D. Arakawa, S. Igarashi, J. Kishiro, K. Koba, K. Takayama \\ High Energy Accelerator Research Organization (KEK), 1-1 Oho, Tsukuba, Ibaraki 305 Japan
}

\begin{abstract}
Towards the intensity up-grade of the KEK-12GeV-PS, the beam loss at the beginning of acceleration should be removed. The loss observed around $80 \mathrm{~ms}$ after the beginning of acceleration results from the horizontal head-tail instability. The instability occurs due to a large change in the chromaticity produced by the sextupole field induced in the beam-pipe of the dipole magnets. The horizontal chromaticity moves into a positive region just around $80 \mathrm{~ms}$ after the beginning of acceleration. The longitudinal mode 0,1 and 2 have been observed.
\end{abstract}

\section{INTRODUCTION}

The operating point, $\left(\nu_{x}, \nu_{y}\right)$, has been changed from (7.12, $7.21)$ to $(7.12,5.21)$ since January 1996 , expecting a reduction of the space-charge induced effects[1]. After the modification, the beam loss emerges around $80 \mathrm{~ms}$ after the beginning of acceleration ( P2 ). Chromaticity control using one of the two sextupole families has been the most effective cure to suppress the beam loss. The observation using the position monitor revealed that the instability is caused by a horizontal coherent dipole motion, which was identified as the Head-Tail Instability ( HTI ) of longitudinal mode, mainly, $\ell=0$. The beam loss around $80 \mathrm{~ms}$ after P2 has almost vanished by properly exciting sextupoles.

\section{EXPERIMENTAL RESULTS}

The typical profile of the horizontal coherent dipole oscillation is shown in Fig.1. In Fig.2 the growth of the oscillation is shown in the lower trace and the beam intensity in the upper trace. The signals are sampled at the timing of the bunch center, $5 \mathrm{k}$ samples per division. The start point of the trace is $50 \mathrm{~ms}$ after P2. The instabilities occur repeatedly even decreasing beam intensity. Around $80 \mathrm{~ms}$ from $\mathrm{P} 2$ the mode $\ell=0$ was mostly observed. On the other hand the mode $\ell=1,2$ or some mixtures were observed at the neighbour of the above time region.

It is well known that the head-tail motion of mode $\ell=$ 0 is unstable in the positive chromaticity region under the transition energy and has no instability threshold in the beam intensity[2]. The chromaticity is controlled by two families of correction sextupoles. Their main tasks are to correct the chromaticity after the transition energy because the sign of the phase slippage factor $\eta=\alpha-1 / \gamma^{2}$ changes, and to correct during slow beam extraction for controlling the betatron tune spread. The power supplies are unipolar and not stable near zero current. Therefore they are excited by a current more than about $2 \mathrm{~A}$.

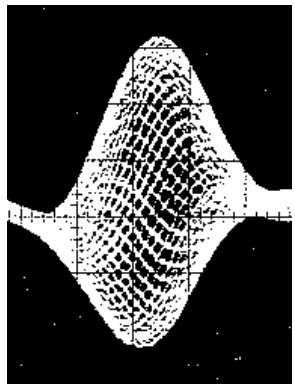

Figure 1: Horizontal coherent oscillation.

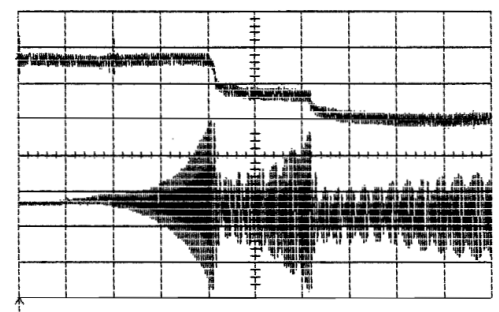

Figure 2: Growth of the coherent oscillation. Upper trace: beam intensity $\left(10^{11}\right.$ protons $/$ div $)$, lower trace: coherent oscillation, abscissa: $5 \mathrm{k}$ samples/div.

Near injection energy, there are some other contributions to the chromaticity other than natural chromaticity and the one from the correction sextupoles, which are not negligible small [3]: a remnant of the correction sextupoles, a sextupole component of the dipole and quadrupole magnets, and an eddy current in the dipole beam-pipe. Calculated contributions are $\xi_{x \text { natural }}=-7.95, \Delta \xi_{\text {x remnant }}=$ $1.90, \Delta \xi_{x d i}=-1.63, \Delta \xi_{\text {xquad }}=0.75$ at the injection energy, $\Delta \xi_{x \text { eddy }}=6.19$ at $100 \mathrm{~ms}$ after P2 and in addition from the correction sextupoles $\Delta \xi_{\text {x sext }}=3.2$ at the injection energy. Here the chromaticity is defined as $\xi=\Delta \nu /(\Delta p / p)$ and the eddy current contribution is estimated using a two-parallel-plate approximation for the beam pipe [4]: $\partial^{2} B_{y} / \partial x^{2}=2 \mu_{0} \sigma(h / g) \dot{B}$, where $\mu_{0}$ is the permiability of the vacuum, $\sigma$ the conductivity of the beam pipe, $h$ the thickness of the beam pipe and $g$ the gap height of the magnet.

Taking the energy dependence of the chromaticity into account as

$$
\begin{gathered}
\xi_{x}(t)=\xi_{\text {x natural }}+\Delta \xi_{x \text { sext }}+\Delta \xi_{x d i}+\Delta \xi_{x \text { quad }}+ \\
\Delta \xi_{\text {x remnant }} \frac{B(0 m s)}{B(t)}+\Delta \xi_{\text {xeddy }} \frac{\dot{B}(t) / B(t)}{\dot{B}(100 m s) / B(100 m s)}
\end{gathered}
$$

we obtain the time evolution of the chromaticity, which is shown with the measured chromaticity in Fig.3. The solid 
line and the filled circles are the calculated and measured results, respectively, for the operation in which the beam loss occurs due to the uncorrected chromaticity at (7.12, 5.21). The calculation including the eddy current effect well agrees with the measured result and well explains the beam loss around $80 \mathrm{~ms}$ from $\mathrm{P} 2$, when the chromaticity moves into the positive region around $80 \mathrm{~ms}$ from $\mathrm{P} 2$.

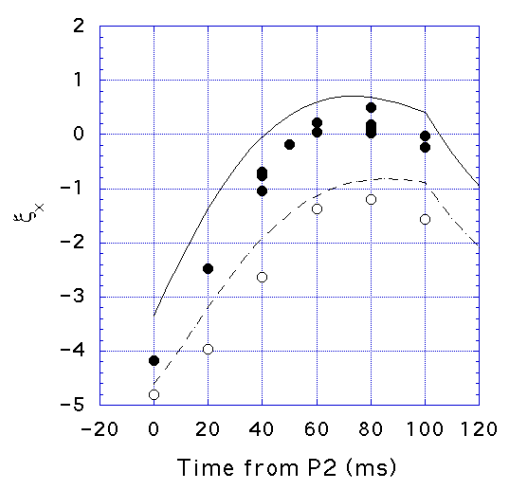

Figure 3: Horizontal chromaticity after the acceleration start.

The natural chromaticity is $\xi_{x}=-9.22$ for the previous operating point, $(7.12,7.21)$. Now we can understand the reason why the head-tail instability emerged when changing the operating point. The increase of the natural chromaticity, $\sim 1.3$, caused zero crossing of the horizontal chromaticity around $80 \mathrm{~ms}$ after $\mathrm{P} 2$ and forced the horizontal coherent motion, mainly, of mode 0 unstable and resulted in the beam loss.

Since the incoherent space charge tune shift has no effect for the transverse dipole mode, we need some other stabilizing effects. The cure has been achieved by the defocusing sextupole family $\left(S_{D}\right)$ because of the power supply polarity. Corresponding chromaticities are plotted in Fig.3 by the broken line (calculated result) and the open circles (measured result).

Another cure was tried using an octupole magnet. The instability was suppressed by Landau damping at the tune spread of $\sim 100 \epsilon_{x}$ (Fig.4), which is consistent with the simulation result described below.

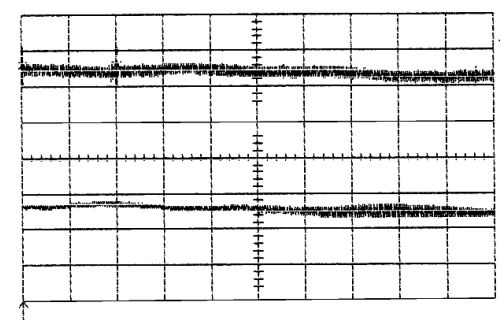

Figure 4: Damping of the coherent oscillation by the octupole magnet. Upper trace: beam intensity $\left(10^{11}\right.$ protons $/$ div $)$, lower trace: coherent oscillation, abscissa: $5 \mathrm{k}$ samples/div.

\section{HTI SIMULATION AND DYNAMIC APERTURE}

To understand the qualitative feature of the observed HTI a multi-particle simulation code has been developed. In the code the continuous focusing model was employed for the purpose of saving a computation time, transverse wakes were assumed to be constant along the ring, and the synchrotron oscillation was taken account of in the form of changes in energy and phase every turn. Accordingly the change in betatron tune resulted from the finite value of chromaticity was given every turn. Meanwhile, effects due to an octupole magnet were included as a delta-function like kick which was near the real situation in the experiment.

The product of constant wake and beam intensity is a key parameter to determine the instability. Unfortunately the magnitude of wake in the KEK-PS was unknown. Here the product was dealt as a free parameter. To elucidate effects of the chromaticity on the growth of the HTI for different modes, the simulation results are shown in Fig.5. As expected from the theoretical prediction, the mode with a large positive growth has been confirmed to vary as a function of the chromaticity. General features of Landaudamping due to the nonlinear tune-spread have been simulated assuming octupole fields. The maximum size of the oscillation amplitude of beam-centroid is depicted as a function of turn-number in Fig.6, where its saturation and succeeding damping are found. These are typical features of Landau Damping.
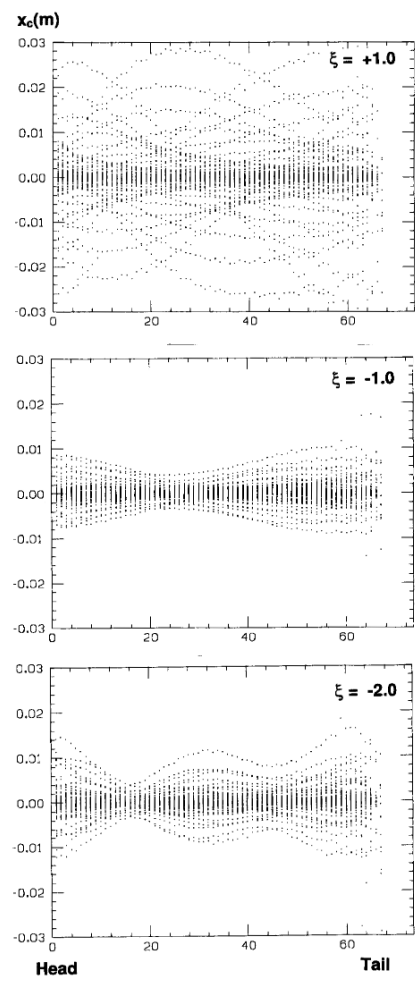

Figure 5: Motion of beam-centroid for different magnitude of the chromaticity. 


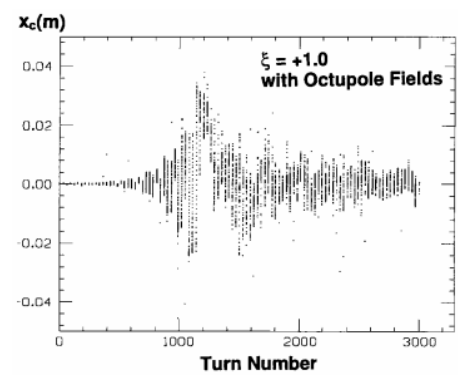

Figure 6: Simulation of Landau damping with octupole magnet fields.

There are some methods to cure the HTI. As mentioned above one is to control chromaticity in a delicate manner by the correction sextupoles and another is to stabilize by Landau damping using octupoles. The excitation of these magnets may reduce the dynamic aperture. Therefore we have examined the dynamic aperture, developing a simulation code by including nonlinear elements, such as sextupole and octupole magnets. A phase space vector $\left(x, x^{\prime}\right)$ is multiplied by the transfer matrix in the linear field components and $x^{\prime}$ is shifted at each nonlinear field component using the thin lens approximation. Poincaré map, $\left(x, \alpha x+\beta x^{\prime}\right)$ is plotted in Fig. 7 when 16 sextupole magnets were considered to be in an operating condition of suppressing HTI and the induced sextupole field component in the dipole beam-pipe are considered to be localized at the center of the dipole magnets.

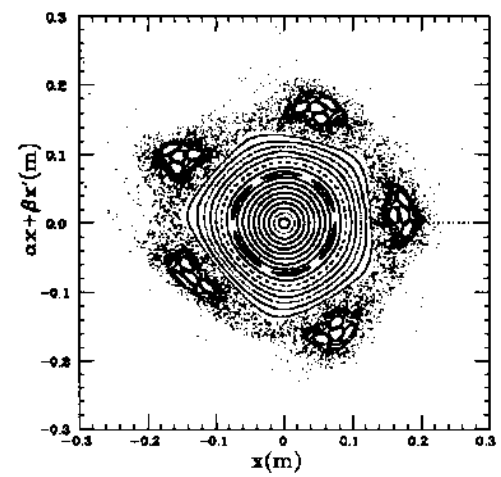

Figure 7: Dynamic aperture with the sextupole excitation and the eddy current.

Besides the correction by the sextupole magnets, Poincaré map is depicted considering the single octupole excitation with the same configuration of the experiment. In this case the dynamic aperture is greatly reduced as shown in Fig.8. More larger dynamic aperture will be obtained by the symmetrically distributed octupole magnets.

\section{SUMMARY}

Summarizing the present operation at the beam intensity of $\sim 5 \times 10^{12}$ protons per pulse ( 9 bunches), the instability is almost suppressed by exciting the defocusing sextupole

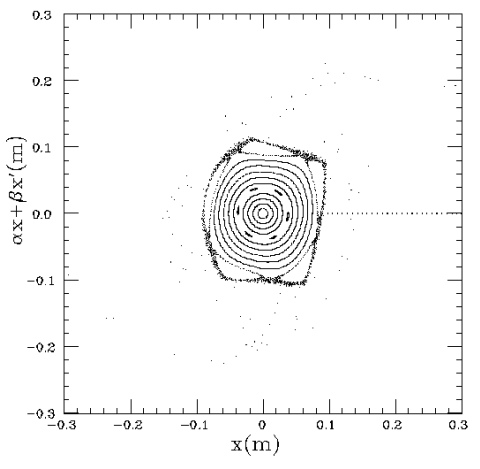

Figure 8: Dynamic aperture with the octupole excitation and the eddy current.

family $\left(S_{D}\right)$. The observed behavior of the HTI is well explained by the simulation and the existing theory.

We are further investigating the HTI, which comprises single- and multi-bunch effects, and will optimize the cure.

\section{ACKNOWLEDGEMENTS}

The authors wish to thank KEK-PS members for their interest and continual support through this work. They wish to thank Prof. I. Yamane and Prof. H. Sato for calling their attention to the present problem. They also wish to thank Dr. M. Shirakata and Dr. M. Uota for helping in the experiment and giving usefull comments.

\section{REFERENCES}

[1] S. Machida and Y. Shoji, AIP Conf. Proc. 377 (1995) p.160, S. Machida, Nucl. Instr. Meth. A 384 (1997) 316. and Y. Shoji et. al., these proceedings.

[2] A. Chao, "Physics of collective beam instabilities in high energy accelerators", John Wiley \& Sons, Inc., 1993 and the references there in.

[3] A. Ando, et. al.: IEEE Trans. on Nucl. Sci. NS-24, No.3 (1977) p.1530 , A. Ando, et. al.: Proc. of MT-5 (1975) p.63, Y. Shoji: KEK Internal Report, SR-316 (1995) (in Japanese).

[4] D. A. Edwards and M. J. Syphers, "An Introduction to the Physics of High Energy Accelerators", John Wiley \& Sons, Inc., 1993. 